\title{
Peritonitis Following Pre-Pyloric Ulcer Perforation: A Case Report
}

\section{Seyed Farshad Heidari*}

Department of Emergency Medicine, Emam Khomeini Hospital, Medical Faculty, Mazandaran University of Medical Sciences, Sari, Iran.

*Corresponding author: Seyed Farshad Heidari, Department of Emergency Medicine, Emam Khomeini Hospital, Medical Faculty, Mazandaran University of Medical Sciences, Sari, Iran. Tel: +981133355080; E-mail: s.f.heidari@gmail.com

Received: June 01, 2017; Accepted: June 26, 2017; Published: July 04, 2017

Citation: Heidari SF (2017) Peritonitis Following Pre-Pyloric Ulcer Perforation: A Case Report. Ann Clin Lab Res 5: 2

\section{Abstract}

Peritonitis due to peptic ulcer perforation is a surgical emergency with a high risk of mortality and morbidity. A case was presented here, that is a 19 -year-old man who underwent an emergency laparotomy for peritonitis caused by perforation of peptic ulcer without risk factor for PUD. The ulcer was located on the pre-pyloric region, close to the lesser curvature. This case report highlights the importance of maintaining a high clinical suspicion of a perforated peptic ulcer in patients presenting with an acute abdomen without risk factor for peptic ulcer.

Keywords: Peritonitis; Peptic ulcer; Perforation; Case report

\section{Introduction}

Perforation of the gastrointestinal tract is one of the lifethreatening causes of acute abdomen that presenting with acute abdominal pain and severe distress of the patient. In one third of patients, the presentation is less obvious, resulting in significant delays in diagnosis of peritonitis. Peritonitis due to perforation of the $\mathrm{GI}$ tract is a common surgical emergency allover the world [1], that occurs in about $2 \%$ to $14 \%$ of patients with active ulcer disease [2,3]. Surgical treatment of perforation peritonitis strongly recommended and included combination of improved surgical technique, anti-microbial therapy and intensive care support has improved the outcome of such cases [4]. The aim of this case report is presentation of a case with gastric perforation without apparent manifestation and risk factor for peptic ulcer disease (PUD).

\section{Case report}

A 19-year-old man presented to the emergency department with a history of abdominal pain, nausea and anorexia from 4days ago. Abdominal pain was periodic during this period. There was history of sudden pain in the upper abdomen on the 4th day that was persistent. Abdominal pain was in severe grade and not relieved by medication. His job was soldier that was started from one-year-ago. In past medical history, the patient had significant history of epigastric pain from the last 12-months without diagnostic evaluation for its cause. He has no history of smoking or drinking alcohol. Drug history was negative. On admission, he had the normal appearance with blood pressure $110 / 70 \mathrm{mmHg}$, pulse rate 85 beats per minute and temperature of $37.1^{\circ} \mathrm{C}$. Abdominal examination revealed generalized tenderness. Also, guarding and rigidity were present and occasional bowel sounds were also noted. Other examinations were normal. Plain upright chest $\mathrm{X}$-ray demonstrated excessive free gas under both hemi-diaphragms that has been showed in Figure 1. Preoperative laboratory investigations were in normal range with $\mathrm{Hb}(15 \mathrm{~g} / \mathrm{dL})$, renal function test (serum urea, $35 \mathrm{mg} / \mathrm{L}$; serum creatinine, 1.1 $\mathrm{mg} / \mathrm{dL}$ ) and electrolytes (serum sodium, $140 \mathrm{meq} / \mathrm{L}$, serum potassium, $4.2 \mathrm{meq} / \mathrm{L}$ ). The broad-spectrum antibiotics, a combination of injectable third generation cephalosporin and metronidazole, were administered for patient. After initial resuscitation (placement of intravenous lines and nasogastric tube followed by adequate administration of fluids), the patient underwent an emergency exploratory laparotomy. During laparotomy, one gastric perforation was identified in the pre-pyloric region, close to the lesser curvature, that the perforations were repaired. Post-operative period was smooth. Patient was discharged on the 7th post-operative day.

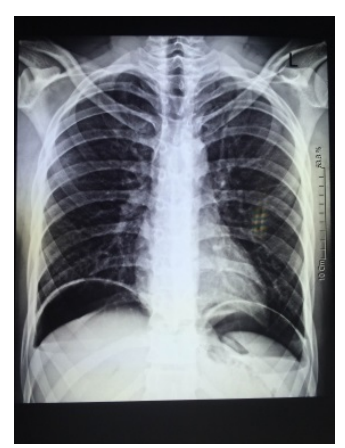

Figure 1 Chest $x$ ray (upright) in patient with perforated prepyloric ulcer. Plain upright chest $x$-ray demonstrated excessive free gas under both hemi-diaphragms.

\section{Discussion}

Peptic ulcer disease (PUD) is associated with potential lifethreatening complications, including bleeding, perforation, penetration and obstruction. Perforation is the second most 
common complication [5], that often present with acute abdomen. Although perforated peptic ulcer frequently is considered a rather benign condition, but it is a lifethreatening complication that carries high risk for morbidity and mortality [6]. The number of patients admitted for emergency surgery after peptic ulcer perforation has not undergone a similar decline, and peptic ulcer perforation remains a substantial healthcare problem [7]. Peptic perforation is most commonly present in the first part of the duodenum ( $35 \%$ to $65 \%$ ), with $25 \%$ to $45 \%$ located in the pylorus, and $5 \%$ to $25 \%$ in the stomach [1]. The etiological factors responsible for peptic perforation vary depending upon sociodemographic factors [8-10]. GI perforation occurs often secondarily to an underline disease, particularly, acute diverticulitis, acute appendicitis, gastric and duodenal ulcers, ischemic colitis and inflammatory bowel disease (IBD) [8]. The factors that participate frequently in occurrence of peptic perforation are helicobacter pylori infection and chronic use of NSAIDs [9]. Other factors influencing in Gl perforation generally occurs as a result of stress, excessive smoking, and consumption of alcohol or coffee [10]. The best surgical option for these patients is simple closure with omental patch. It is the easiest, quickest and safest operation, and can be applied to all situations by every surgeon. In this patient who was introduced here, as regards the symptoms of perforation were not evident, the patient presented to hospital with delayed. Also as respects the patient had no risk factors for perforation. Peptic ulcer diseases even cases are complicated when presented in young age has many causes that must be examined include zollinger-ellison disease, behcet's disease, drug addiction, lymphoma and etc.

\section{Conclusion}

Perforated peptic ulcer is not rare and it is relatively simple to have the diagnosis that needs to immediate treatment.

\section{References}

1. Bulut OB, Rasmussen C, Fischer A (1996) Acute surgical treatment of complicated peptic ulcers with special reference to the elderly. World J Surg 20: 574-577.

2. Bertleff MJ, Lange JF (2010) Perforated peptic ulcer disease: A review of history and treatment. Dig Surg 27: 161-169

3. Lau JY, Sung J, Hill C, Henderson C, Howden CW, et al. (2011) Systematic review of the epidemiology of complicated peptic ulcer disease: incidence, recurrence, risk factors and mortality. Digestion 84: 102-113.

4. Bosscha K, Van Vroonhoven TJ, Van der Werken C (1999) Surgical management of severe secondary peritonitis. $\mathrm{Br} J$ Surg 86: 1371-1377.

5. Milosavljevic T, Kostic-Milosavljevic M, Jovanovic I, Krstić M (2011) Complications of peptic ulcer disease. Dig Dis 29: 491493.

6. Bas G, Eryilmaz R, Okan I, Sahin M (2008) Risk factors of morbidity and mortality in patients with perforated peptic ulcer. Acta Chir Belg 108: 424-427.

7. Kim JM, Jeong SH, Lee YJ, Park ST, Choi SK, et al. (2012) Analysis of risk factors for postoperative morbidity in perforated peptic ulcer. J Gastric Cancer 12: 26-35.

8. Wei SC, Tan YY, Weng MT, Lai LC, Hsiao JH, et al. (2014) SLCO3A1, a Novel Crohn's disease-associated Gene, regulates NF-kB activity and associates with intestinal perforation. PLoS One 9: e100515.

9. Hopkins RJ, Girardi LS, Turney EA. (1996) Relationship between helicobacter pylori eradication and reduced duodenal and gastric ulcer recurrence: A review. Gastroenterol 110: 1244-1252.

10. Kim Y, Yokoyama S, Watari J, Hori K, Yamasaki T, et al. (2012) Endoscopic and clinical features of gastric ulcers in Japanese patients with or without helicobacter pylori infection who were using NSAIDs or low dose aspirin. J Gastroenterol 47: 904-911. 